Meta

Journal des traducteurs

Translators' Journal

\title{
Lexique anglais-français de la radiotéléphonique maritime
}

\section{Pierre Biron}

Volume 25, numéro 3, septembre 1980

URI : https://id.erudit.org/iderudit/002084ar

DOI : https://doi.org/10.7202/002084ar

Aller au sommaire du numéro

Éditeur(s)

Les Presses de l'Université de Montréal

ISSN

0026-0452 (imprimé)

1492-1421 (numérique)

Découvrir la revue

Citer cet article

Biron, P. (1980). Lexique anglais-français de la radiotéléphonique maritime. Meta, 25(3), 364-366. https://doi.org/10.7202/002084ar d'utilisation que vous pouvez consulter en ligne.

https://apropos.erudit.org/fr/usagers/politique-dutilisation/ 


\section{LEXIQUE ANGLAIS-FRANÇAIS DE LA RADIOTÉLÉPHONIQUE} MARITIME

Acknowledge

Acknowledgement of receipt

Affirmative

Aircraft

All stations

Bad Readability

Break

Call sign

Call to all stations

Calling

Cancellation of message

Channel

Confirm

Distress communication

Excellent Readability

Fair Readability

For

From
Veuillez accuser réception!

Accusé de réception

Affirmatif

Aéronef

Toutes les stations

Mauvais

Séparatif

Indicatif d'appel

Appel à toutes les stations

Appel

Annulation de message

Voie; canal

Confirmez

Communication en cas de détresse

Excellent

Passable

À destination de

En provenance de

5. Le Robert et Collins, dictionnaire français/anglais, anglais/français, 1978.

6. Harrap's Standard French and English Dictionary, part two, 1966.

7. «On Linguistic Aspects of Translation», in R. A. Brower, On Translation, Harvard University Press, 1959, p. 236. 
General Radiotelephone Operator's Certificate

Go ahead

Good Readability

How do you read

I say again!

Imposition of silence

International Civil Aviation

Organization

International Telecommunications Union

Maritime Mobile Service

Mayday [From French m'aider]

Mayday relay

Message addressed to

a specific station

Multiple station call

Negative

Operator's certificate

Out

Over

Pan pan

Phonetic spelling alphabet

Poor Readability

Procedure word

Radio Aids to Maritime Navigation

Radio $\log$

Readability scale

Replying

Restricted Radiotelephone

Operator's Certificate

Roger

Roger number

Say again

Say again to

Say again all after

Say again all before

Say again your call sign

Search and Rescue Organization

Security

Ship

Silence distress

Silence mayday

Simple station call

Spoken numerals
Certificat général de radiotéléphoniste

Continuez

Bon

Comment recevez-vous

Je dis de nouveau

Imposition du silence

Organisation de l'aviation civile internationale

Union internationale des télécommunications

Service mobile maritime

Mayday

Mayday relay

Message adressé à

une station déterminée

Appel à plusieurs stations

Négatif

Certificat d'opérateur

Terminé

Répondez

Panne Panne

Épellation en alphabet phonétique

Médiocre

Expression commentionnelle

Aides radio à la navigation maritime

Journal radio

Échelle d'intelligibilité

Réponse

Certificat restreint de radiotéléphoniste

Roger

Roger numéro

Dites de nouveau

Dites de nouveau tout entre et

Dites de nouveau tout après

Dites de nouveau tout avant

Dites de nouveau votre indicatif d'appel

Organisation de recherches et de sauvetage

Sécurité

Navire

Silence détresse

Silence mayday

Appel simple

Chiffres parlés 
Station called

Station calling!

Testing

That is correct

This is

Urgency communication

Urgency signal

Verify

Wilco

Words twice
Station appelée

Station appelante

Essais

C'est exact

Ici

Communication d'urgence

Signal d'urgence

Vérifiez

Wilco

Chaque mot deux fois

Pierre Biron 